\title{
A Discrete Mathematical Modeling of the Influence of Alcohol Treatment Centers on the Drinking Dynamics Using Optimal Control
}

\author{
Bouchaib Khajji $\left(\mathbb{D},{ }^{1}\right.$ Abderrahim Labzai ${ }^{(D},{ }^{1}$ Abdelfatah Kouidere ${ }^{(D)},{ }^{1}$ Omar Balatif ${ }^{(D)}{ }^{2}$ \\ and Mostafa Rachik ${ }^{1}$ \\ ${ }^{1}$ Laboratory of Analysis Modeling and Simulation, Department of Mathematics and Computer Science, \\ Faculty of Sciences Ben M'Sik, Hassan II University, Sidi Othman, Casablanca, Morocco \\ ${ }^{2}$ Mathematical Engineering Team (INMA), Laboratory of Dynamical Systems, Department of Mathematics, \\ Faculty of Sciences El Jadida, Chouaib Doukkali University, El Jadida, Morocco
}

Correspondence should be addressed to Bouchaib Khajji; khajjibouchaib@gmail.com

Received 12 November 2019; Revised 5 January 2020; Accepted 23 January 2020; Published 19 February 2020

Academic Editor: Fernando Simões

Copyright (c) 2020 Bouchaib Khajji et al. This is an open access article distributed under the Creative Commons Attribution License, which permits unrestricted use, distribution, and reproduction in any medium, provided the original work is properly cited.

\begin{abstract}
In this paper, we propose a discrete mathematical model that describes the interaction between the classes of drinkers, namely, potential drinkers $(P)$, moderate drinkers $(M)$, heavy drinkers $(H)$, poor heavy drinkers $\left(T^{p}\right)$, rich heavy drinkers $\left(T^{r}\right)$, and quitters of drinking $(Q)$. We also focus on the importance of treatment within addiction treatment centers aiming to find the optimal strategies to minimize the number of drinkers and maximize the number of heavy drinkers who join addiction treatment centers. We use three controls which represent awareness programs through media and education for the potential drinkers, efforts to encourage the heavy drinkers to join addiction treatment centers, and psychological support with follow-up for the individuals who quit drinking. We use Pontryagin's maximum principle in discrete time to characterize these optimal controls. The resulting optimality system is solved numerically by Matlab. Consequently, the obtained results confirm the performance of the optimization strategy.
\end{abstract}

\section{Introduction}

Addiction is a social phenomenon and a psychological scourge that all societies suffer from. It affects all social classes and all people with different educational levels and age groups. There are many types of addiction such as addiction to smoking, addiction to eating, sugar addiction, addiction to video games, addiction to social networking sites, mobile phone addiction, addiction to sleep, and addiction to drinking. In this work, we will investigate the phenomenon of addiction to drinking alcohol which is a problem that has received the attention of several researchers and scholars in many fields including psychology, sociology, psychiatry, and mathematics, in an attempt to highlight the reasons behind this phenomenon and identify effective methods of prevention and treatment to this problem.

According to the World Health Organization (WHO) report in 2016, alcohol addiction causes the death of more than 3 million people annually, which represents about 6 percent of all deaths worldwide. In spite of its fatal harm, global consumption of alcohol is expected to increase in the next 10 years [1]. Currently, about 2.3 billion people are drinking alcohol, including 237 million men and 46 million women around the world who drink excessively or badly according to the World Health Organization in its Health Report in 2018 [1]. In some places, drinking alcohol is more common such as in Europe and the Americas which come at the top of the list followed by Russia according to the same organization. Alcohol-induced disorders exist in richer 
countries more than the poor ones. In some countries like Russia, the annual consumption of alcohol (measured in liters of pure ethanol) per capita is decreasing yearly as it moved from 18.7 liters in 2005 to 11.7 liters in 2016. This "dramatic decrease" can be explained by the implementation of measures recommended by WHO such as the prohibition of the sale of alcoholic beverages at service stations. Yet, WHO in its report expects that the global alcohol consumption will rise over the next decade, especially in the Southeast Asia, Western Pacific, and the Americas, and this increase will surely affect the number of deaths all over the world [1].

In Morocco, according to the WHO Global Status Report on Alcohol and Health 2018, Moroccans are following the global trend in alcohol consumption. Thus, we note that the consumption of alcohol has decreased from 1.8 liter per individual in 2010 to 1.1 liter in 2016 for men and 0.2 liter in 2010 to 0.1 liter in 2016 for women [2].

Addiction to drinking alcohol has many health and social consequences including chronic diseases, gastrointestinal disorders, increased risk of pancreatitis and ulcers, severe damage to the liver, damage to the brain and the nervous system, muddled thinking and loss of memory, harmful hormonal performance, increased risk of heart disease and stroke, family problems, work problems, social marginalization, poverty, road accidents, and undesirable morals [1].

Alcohol can be prevented through a number of measures such as regulating and limiting its sales, increasing its cost, and offering inexpensive treatment. The treatment can pass through several steps due to medical issues that may arise during the period of quitting alcohol; therefore, careful control must be exercised on the drinkers to help them stop alcohol consumption. Some medications can be given to the addict while he/she is in a health facility or from time to time while the person lives within the community under close supervision. Psychological disorders or other addictions can complicate the treatment, so, after quitting alcohol, support and follow-up are used in group therapy or support groups to help the person quit drinking again. The most common types of support are given by psychiatrists and alcoholics who gave up alcohol also by giving medicaments such as Acamprosate, Disulfiram, or Naltrexone.

Some researchers in mathematics draw a comparison between the spread of the drinking phenomenon and the spread of infectious diseases. Accordingly, several mathematicians did a lot of work in order to understand the dynamics of drinking and reduce its harm on the drinker and society as well as minimizing the number of addicted drinkers. For example, Huo and Wang [3] developed a nonlinear mathematical model with the effect of awareness programs on the binge drinking where they show that awareness programs are an effective measure in reducing alcohol problems. Ma et al. [4] modeled alcoholism as a contagious disease and used an optimal control to study their mathematical model with awareness programs and time delay. Wang et al. [5] proposed and analyzed a nonlinear alcoholism model and used optimal control for the purpose of hindering interaction between susceptible individuals and infected individuals. Huo et al. [6] proposed a new social epidemic model to depict alcoholism with media coverage which was proven to be an effective way in pushing people to quit drinking. Huo and Song [7] divided heavy drinkers in their study into two types: those who confess drinking and those who do not, and they proposed a two-stage model for binge drinking problem taking into consideration the transition of drinkers from the class of susceptible individuals towards the class of admitting drinkers. Adu et al. [8] used a nonlinear SHTR mathematical model to study the dynamics of drinking epidemic; they divided their population into four classes: nondrinkers $(S)$, heavy drinkers $(H)$, drinkers receiving treatment $(T)$, and recovered drinkers $(R)$. They discussed the existence and stability of drinking-free and endemic equilibrium. Other mathematical models have also been widely used to study this phenomenon (for example, [5, 9-12]).

In addition, most of these previous researches have focused on continuous-time modeling. In this research, we will adopt the discrete-time modeling as the statistical data are collected at discrete time (day, week, month, and year) and the treatment and vaccination of some patients are done in discrete time. So, it is more direct, more convenient, and more accurate to describe the phenomena by using the discretetime modeling than the continuous-time modeling and the use of discrete-time models may avoid some mathematical complexities such as the choice of a function space and regularity of the solution. Hence, difference equations appear as a more natural way to describe the epidemic models. Moreover, numerical solutions of differential equations use discretization and this encourages us to employ difference equations directly. The numerical exploration of discrete-time models is rather straightforward and therefore can be easily implemented by nonmathematicians.

Besides these works, we will study the dynamics of a mathematical alcohol model $P M H T^{r} T^{p} Q$ which contains the following additions:

(i) A discrete-time mathematical modeling

(ii) A compartment $T^{p}$ that represents the number of the poor heavy drinkers who join public addiction treatment centers which may not afford sophisticated equipment and high-quality treatment services-especially in the developing countries-and those individuals who do not have the financial capacity to go to private centers

(iii) A compartment $T^{r}$ that represents the number of the rich heavy drinkers who join private addiction treatment centers which have special facilities that provide advanced treatment and individuals who have sufficient financial capacity to join these centers

(iv) The death rate induced by the heavy drinkers $\delta$

The drinkers classes of this model are divided into six compartments: potential drinkers $(P)$, moderate drinkers $(M)$, heavy drinkers $(H)$, the rich heavy drinkers who join private addiction treatment centers $\left(T^{r}\right)$, the poor heavy drinkers who join public addiction treatment centers $\left(T^{p}\right)$, 
and quitters of drinking $(Q)$. Throughout this research, we seek to find the optimal strategies to minimize the number of drinkers and maximize the number of rich and poor heavy drinkers who join addiction treatment centers.

In order to achieve this purpose, we use optimal control strategies associated with three types of controls: the first represents awareness programs for potential drinkers, the second is the effort to encourage the rich to go to private treatment centers and the poor heavy drinkers to go to public treatment centers, and the third represents follow-up and the psychological support for temporary quitters of drinking.

The paper is organized as follows. In Section 3, we present our $P M H T^{r} T^{P} Q$ discrete mathematical model that describes the classes of drinkers. In Sections 4 and 5, we present the optimal control problem for the proposed model where we give some results concerning the existence of the optimal controls and we characterize these optimal controls using Pontryagin's maximum principle in discrete time. Numerical simulations are given in Section 6. Finally, we conclude the paper in Section 6.

\section{Model Formulation}

We propose a discrete model $P M H T^{r} T^{p} Q$ to describe the dynamics of population and the transmission of drinking. The population is divided into six compartments denoted by $P, M, H, T^{r}, T^{p}$, and $Q$.

The graphical representation of the proposed model is shown in Figure 1.

The mathematical representation of the model consists of a system of nonlinear difference equations:

$$
\left\{\begin{array}{l}
P_{k+1}=b-\beta_{1} \frac{P_{k} M_{k}}{N_{k}}+(1-\mu) P_{k}, \\
M_{k+1}=\beta_{1} \frac{P_{k} M_{k}}{N_{k}}+\theta Q_{k}+\left(1-\mu-\beta_{2}\right) M_{k}, \\
H_{k+1}=\beta_{2} M_{k}+\left(1-\mu-\delta-\alpha_{1}-\alpha_{2}-\alpha_{3}\right) H_{k}, \\
T_{k+1}^{r}=\alpha_{1} H_{k}+\left(1-\mu-\gamma_{1}\right) T_{k}^{r}, \\
T_{k+1}^{p}=\alpha_{2} H_{k}+\left(1-\mu-\gamma_{2}\right) T_{k}^{p} \\
Q_{k+1}=\gamma_{1} T_{k}^{r}+\gamma_{2} T_{k}^{p}+\alpha_{3} H_{k}+(1-\mu-\theta) Q_{k},
\end{array}\right.
$$

where $P_{0} \geq 0, M_{0} \geq 0, H_{0} \geq 0, T_{0}^{r} \geq 0, T_{0}^{p} \geq 0$, and $Q_{0} \geq 0$.

The compartment $P$ contains the potential drinkers who represent individuals whose age is over adolescence and adulthood and may become drinkers. This compartment is increased by the recruitment rate denoted by $b$ and decreased by an effective contact with the moderate drinkers at $\beta_{1}$ rate and natural death $\mu$. It is assumed that potential drinkers can acquire drinking behavior and can become moderate drinkers through effective contact with moderate drinkers in some social occasions like weddings, celebrating graduation ceremonies, weekend parties, and end of the year celebration. In other words, it is assumed that the acquisition of a drinking behavior is analogous to acquiring disease infection.

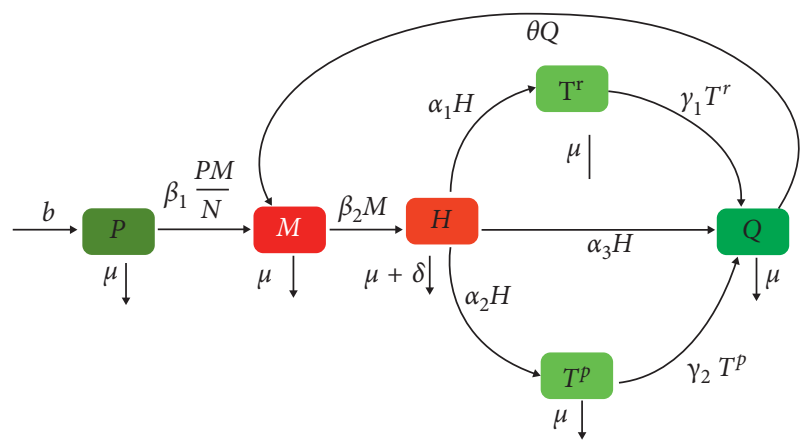

FIgURE 1: Schematic diagram of the six drinking classes in the model.

The compartment $M$ is composed of the moderate drinkers who drink alcohol in a controlled manner during some events and occasions or in a way that is unapparent to their social environment. It is increased by potential drinkers who turn to be moderate drinkers at $\beta_{1}$ rate and those who quit addiction to alcohol temporarily at a rate $\theta$. This compartment is decreased when moderate drinkers become heavy drinkers at a rate $\beta_{2}$ and also by natural death at rate $\mu$.

The compartment $H$ comprises the heavy drinkers. This compartment becomes larger as the number of heavy drinkers increases by the rate $\beta_{2}$ and decreases when some of them give up drinking at a rate $\alpha_{3}$ and decreases also by the rate $\alpha_{1}\left(\alpha_{1}\right.$ is a rate the heavy drinkers join private treatment centers) and decreases also by the rate $\alpha_{2}\left(\alpha_{2}\right.$ is a rate the heavy drinkers join public treatment centers). In addition, this compartment decreases by natural death $\mu$ and due to deaths caused by diseases resulted from excessive alcohol intake at a rate $\delta$.

The compartment $T^{p}$ represents the number of heavy drinkers who join public treatment centers of alcohol addiction which may not provide advanced treatment and that are marked by a shortage of equipment and low-quality services-especially in the developing countries-and it also contains individuals who do not have the financial capacity to join the private centers. This compartment is increased by the rate $\alpha_{2}$ and decreased by the rate $\gamma_{2}$ which represents individuals who have been treated in the public treatment centers and also by natural death at rate $\mu$.

The compartment $T^{r}$ contains the number of heavy drinkers who take advantage of their financial potentials to join private treatment centers of alcohol addiction that are very often well-equipped and provide good-quality services. This compartment is increased by the rate $\alpha_{1}$ and decreased by the rate $\gamma_{1}$ which represents individuals who have been treated in the private treatment centers and also by natural death at rate $\mu$.

The compartment $Q$ encompasses the individuals who quit drinking temporarily and permanently. It is increased with the recruitment of individuals who have been treated in treatment centers of alcohol addiction at rates $\gamma_{1}$ and $\gamma_{2}$. It also increases at the rate $\alpha_{3}$ by those who quit alcohol without resorting to treatment centers and decreases at the rate $\mu$ due to natural deaths and at rate $\theta$ (which represents drinkers who quit drinking temporarily and revert back to moderate drinking). We note that $\theta Q_{k}$ is the proportion of 
the temporary quitters of drinking who moved to moderate drinking at time step $k$.

The total population size at time $k$ is denoted by $N_{k}$ with $N_{k}=P_{k}+M_{k}+H_{k}+T_{k}^{r}+T_{k}^{p}+Q_{k}$, and it is supposed to be constant.

\section{The Optimal Control Problem}

The objective of the proposed control strategy is to minimize the number of heavy drinkers $(H)$ and maximize the number of the rich heavy drinkers $\left(T^{r}\right)$ and the poor heavy drinkers $\left(T^{p}\right)$, who join private or public treatment centers of alcohol addiction, and the number of the permanent quitters of drinking $(Q)$ in the community during the time steps $k=0$ to $k=T$. The cost spent in the awareness programs is also to be minimized.

In order to achieve these objectives, we introduce three control variables. The first control $u_{1}$ represents the effort of the awareness programs to protect the potential drinkers not to be drinkers. The second control $u_{2}$ measures the effort made to make the heavy drinkers know private and public treatment centers of alcohol addiction and to encourage them to join those centers. We note that the control function $\epsilon u_{2}$ represents the fraction of the heavy drinkers who will be treated in private addiction treatment centers and the fraction $(1-\epsilon) u_{2}$ of those leaving the heavy drinkers class who will receive treatment in public addiction treatment centers. The third control $u_{3}$ represents the effort of the follow-up and the psychological support for the temporary quitters of drinking with a purpose of protecting them to not go back to drinking.

So, the controlled mathematical system is given by the following system of difference equations:

$$
\left\{\begin{array}{l}
P_{k+1}=b-\beta_{1} \frac{P_{k} M_{k}}{N_{k}}+(1-\mu) P_{k}-u_{1, k} P_{k}, \\
M_{k+1}=\beta_{1} \frac{P_{k} M_{k}}{N_{k}}+\left(1-u_{3, k}\right) \theta Q_{k}+\left(1-\mu-\beta_{2}\right) M_{k}, \\
H_{k+1}=\beta_{2} M_{k}+\left(1-\mu-\delta-\alpha_{1}-\alpha_{2}-\alpha_{3}\right) H_{k}-u_{2, k} H_{k}, \\
T_{k+1}^{r}=\alpha_{1} H_{k}+\left(1-\mu-\gamma_{1}\right) T_{k}^{r}+\epsilon u_{2, k} H_{k}, \\
T_{k+1}^{p}=\alpha_{2} H_{k}+\left(1-\mu-\gamma_{2}\right) T_{k}^{p}+(1-\epsilon) u_{2, k} H_{k}, \\
Q_{k+1}=\gamma_{1} T_{k}^{r}+\alpha_{3} H_{k}+\gamma_{2} T_{k}^{p}+(1-\mu) Q_{k}+u_{1, k} P_{k}-\left(1-u_{3, k}\right) \theta Q_{k},
\end{array}\right.
$$

where $P_{0} \geq 0, M_{0} \geq 0, H_{0} \geq 0, T_{0}^{r} \geq 0, T_{0}^{p} \geq 0$, and $Q_{0} \geq 0$.

The optimal control problem to minimize the objective functional is given by

$$
\begin{aligned}
J\left(u_{1}, u_{2}, u_{3}\right)= & H_{T}-T_{T}^{r}-T_{T}^{p}-Q_{T} \\
& +\sum_{k=0}^{T-1}\left(H_{k}-T_{k}^{r}-T_{k}^{p}-Q_{k}\right. \\
& \left.+\frac{A_{k} u_{1, k}^{2}}{2}+\frac{B_{k} u_{2, k}^{2}}{2}+\frac{C_{k} u_{3, k}^{2}}{2}\right),
\end{aligned}
$$

We apply the discrete version of Pontryagin's maximum
principle [13-18]. The key idea is introducing the adjoint
function to attach the system of difference equations to the
objective functional resulting in the formation of a function
called the Hamiltonian. This principle converts the problem

We apply the discrete version of Pontryagin's maximum
principle [13-18]. The key idea is introducing the adjoint
function to attach the system of difference equations to the
objective functional resulting in the formation of a function
called the Hamiltonian. This principle converts the problem

We apply the discrete version of Pontryagin's maximum
principle [13-18]. The key idea is introducing the adjoint
function to attach the system of difference equations to the
objective functional resulting in the formation of a function
called the Hamiltonian. This principle converts the problem

We apply the discrete version of Pontryagin's maximum
principle [13-18]. The key idea is introducing the adjoint
function to attach the system of difference equations to the
objective functional resulting in the formation of a function
called the Hamiltonian. This principle converts the problem

We apply the discrete version of Pontryagin's maximum
principle [13-18]. The key idea is introducing the adjoint
function to attach the system of difference equations to the
objective functional resulting in the formation of a function
called the Hamiltonian. This principle converts the problem

where the parameters $A_{k}>0 ; B_{k}>0$; and $C_{k}>0$ are selected to weigh the relative importance of the cost of awareness programs, encouragement programs, and follow-up and psychological support, respectively.

The aim is to find an optimal control, $u_{1}^{*}, u_{2}^{*}$, and $u_{3}^{*}$, such that

$$
J\left(u_{1}^{*}, u_{2}^{*}, u_{3}^{*}\right)=\min _{\left(u_{1}, u_{2}, u_{3}\right) \in U_{\mathrm{ad}}} J\left(u_{1}, u_{2}, u_{3}\right),
$$

where $U_{\text {ad }}$ is the set of admissible controls defined by

$$
\begin{aligned}
U_{\mathrm{ad}}=\left\{\frac{u_{j}=\left(u_{j, 0}, u_{j, 1}, \ldots, u_{j, T-1}\right)}{0 \leq u_{j \min } \leq u_{j, k} \leq u_{j \max } \leq 1}\right. & \\
& \quad \text { for } j=1,2,3, k=0,1, \ldots, T-1\} .
\end{aligned}
$$

The sufficient condition for the existence of an optimal control $\left(u_{1}^{*}, u_{2}^{*}, u_{3}^{*}\right)$ for problems (2) and (3) comes from the following theorem.

Theorem 1. There exists the optimal control $\left(u_{1}^{*}, u_{2}^{*}, u_{3}^{*}\right)$ such that

$$
J\left(u_{1}^{*}, u_{2}^{*}, u_{3}^{*}\right)=\min _{\left(u_{1}, u_{2}, u_{3}\right) \in U_{\mathrm{ad}}} J\left(u_{1}, u_{2}, u_{3}\right),
$$

subject to the control system (2) with initial conditions.

Proof. Since the coefficients of the state equations are bounded and there are a finite number of time steps, $P=\left(P_{0}, P_{1}, P_{2}, \ldots, P_{T}\right), M=\left(M_{0}, M_{1}, M_{2}, \ldots, M_{T}\right), H=$ $\left(H_{0}, H_{1}, H_{2}, \ldots, H_{T}\right), \quad T^{r}=\left(T_{0}^{r}, T_{1}^{r}, T_{2}^{r}, \ldots, T_{T}^{r}\right), \quad T^{p}=$ $\left(T_{0}^{p}, T_{1}^{p}, T_{2}^{p}, \ldots, T_{T}^{p}\right)$, and $Q=\left(Q_{0}, Q_{1}, Q_{2}, \ldots, Q_{T}\right)$ are uniformly bounded for all $\left(u_{1}, u_{2}, u_{3}\right)$ in the control set $U_{\text {ad }}$; thus $J\left(u_{1}, u_{2}, u_{3}\right)$ is bounded for all $\left(u_{1}, u_{2}, u_{3}\right) \in U_{\text {ad }}$. Since $J\left(u_{1}, u_{2}, u_{3}\right)$ is bounded, $\inf _{\left(u_{1}, u_{2}, u_{3}\right) \in U_{\mathrm{ad}}} J\left(u_{1}, u_{2}, u_{3}\right)$ is finite, and there exists a sequence $\left(u_{1}^{j}, u_{2}^{j}, u_{3}^{j}\right) \in U_{\text {ad }}$ such that $\lim _{j \longrightarrow+\infty}\left(u_{1}^{j}, u_{2}^{j}, u_{3}^{j}\right)=\inf _{\left(u_{1}, u_{2}, u_{3}\right) \in U_{\text {ad }}} J\left(u_{1}, u_{2}, u_{3}\right)$ and corresponding sequences of states $P^{j}, M^{j}, H^{j}, T^{r j}, T^{p j}$, and $Q^{j}$. Since there are a finite number of uniformly bounded sequences, there exist $\left(u_{1}^{*}, u_{2}^{*}, u_{3}^{*}\right) \in U_{\text {ad }}$ and $P^{*}, M^{*}, H^{*}$, $T^{*}, T^{p *}$ and $Q^{*} \in I R^{T+1}$ such that, on a subsequence, $\lim _{j \longrightarrow+\infty}\left(u_{1}^{j}, u_{2}^{j}, u_{3}^{j}\right)=\left(u_{1}^{*}, u_{2}^{*}, u_{3}^{*}\right), \quad \lim _{j \longrightarrow+\infty} P^{j}=P^{*}$, $\lim _{j \longrightarrow+\infty} M^{j}=M^{*}, \quad \lim _{j \longrightarrow+\infty} H^{j}=H^{*}, \quad \lim _{j \longrightarrow+\infty} T^{r j}=$ $T^{r *}, \lim _{j \longrightarrow+\infty} T^{p j}=T^{p *}$, and $\lim _{j \longrightarrow+\infty} Q^{j}=Q^{*}$. Finally, due to the finite dimensional structure of system (2) and the objective function $J\left(u_{1}, u_{2}, u_{3}\right),\left(u_{1}^{*}, u_{2}^{*}, u_{3}^{*}\right)$ is an optimal control with corresponding states $P^{*}, M^{*}, H^{*}, T^{r *}, T^{p *}$, and $Q^{*}$. Therefore $\inf _{\left(u_{1}, u_{2}, u_{3}\right) \in U_{\text {ad }}} J\left(u_{1}, u_{2}, u_{3}\right)$ is achieved.

\section{Characterization of the Optimal Controls}


of finding the control to optimize the objective functional subject to the state difference equation with initial condition to find the control to optimize Hamiltonian pointwise (with respect to the control). by

Now we have the Hamiltonian $\hat{H}$ at time step $k$, defined

$$
\begin{aligned}
\widehat{H}_{k}= & H_{k}-T_{k}^{r}-T_{k}^{p}-Q_{k}+\frac{A_{k} u_{1, k}^{2}}{2}+\frac{B_{k} u_{2, k}^{2}}{2}+\frac{C_{k} u_{3, k}^{2}}{2} \\
& +\sum_{i=1}^{6} \lambda_{i, k+1} f_{i ; k+1},
\end{aligned}
$$

where $f_{i ; k+1}$ is the right-hand side of the system of difference equation (2) of the $i^{\text {th }}$ state variable at time step $k+1$.

Theorem 2. Given an optimal control $\left(u_{1}^{*}, u_{2}^{*}, u_{3}^{*}\right) \in U_{a d}$ and solutions $P_{k}^{*}, M_{k}^{*}, H_{k}^{*}, T_{k}^{r *}, T_{k}^{p *}$, and $Q_{k}^{*}$ of corresponding state system [13], there exist adjoint functions $\lambda_{1}, \lambda_{2}, \lambda_{3}, \lambda_{4}, \lambda_{5}$, and $\lambda_{6}$ satisfying the following equations:

$$
\begin{aligned}
& \lambda_{1, k}=\frac{\partial \hat{H}_{k}}{\partial P_{k}}=\left(\lambda_{2, k+1}-\lambda_{1, k+1}\right) \beta_{1} \frac{M_{k}}{N_{k}}+\left(\lambda_{6, k+1}-\lambda_{1, k+1}\right) u_{1, k}+\lambda_{1, k+1}(1-\mu) \\
& \lambda_{2, k}=\frac{\partial \widehat{H}_{k}}{\partial M_{k}}=\left(\lambda_{2, k+1}-\lambda_{1, k+1}\right) \beta_{1} \frac{P_{k}}{N_{k}}+\beta_{2} \lambda_{3, k+1}+\lambda_{2, k+1}\left(1-\mu-\beta_{2}\right) \\
& \lambda_{3, k}=\frac{\partial \widehat{H}_{k}}{\partial H_{k}}=1+\lambda_{3, k+1}\left(1-\mu-\delta-\alpha_{1}-\alpha_{2}-\alpha_{3}-u_{2, k}\right)+\lambda_{4, k+1}\left(\alpha_{1}+\epsilon u_{2, k}\right)+\lambda_{5, k+1}\left[\alpha_{2}+(1-\epsilon) u_{2, k} H_{k}\right]+\alpha_{3} \lambda_{6, k+1}, \\
& \lambda_{4, k}=\frac{\partial \widehat{H}_{k}}{\partial T_{k}^{r}}=-1+\gamma_{1} \lambda_{6, k+1}+\lambda_{4, k+1}\left(1-\mu-\gamma_{1}\right) \\
& \lambda_{5, k}=\frac{\partial \widehat{H}_{k}}{\partial T_{k}^{p}}=-1+\gamma_{2} \lambda_{6, k+1}+\lambda_{5, k+1}\left(1-\mu-\gamma_{2}\right) \\
& \lambda_{6, k}=\frac{\partial \widehat{H}_{k}}{\partial Q_{k}}=-1+\lambda_{2, k+1}\left(1-u_{3, k}\right) \theta+\lambda_{6, k+1}\left(1-\mu-\theta+\theta u_{3, k}\right)
\end{aligned}
$$

with the transversality conditions at time $T$ :

$$
\begin{aligned}
& \lambda_{1}(T)=0, \\
& \lambda_{2}(T)=0, \\
& \lambda_{3}(T)=1, \\
& \lambda_{4}(T)=-1, \\
& \lambda_{5}(T)=-1, \\
& \lambda_{6}(T)=-1 .
\end{aligned}
$$

$$
\begin{aligned}
& u_{1, k}^{*}=\min \left\{\max \left\{\frac{\left(\lambda_{1, k+1}-\lambda_{6, k+1}\right) P_{k}}{A_{k}}, u_{1 \min }\right\}, u_{1 \max }\right\}, \\
& u_{2, k}^{*}=\min \left\{\max \left\{\frac{\left(\lambda_{3, k+1}-\lambda_{5, k+1}\right) H_{k}+\left(\lambda_{5, k+1}-\lambda_{4, k+1}\right) \epsilon H_{k}}{B_{k}}, u_{2 \min }\right\}, u_{2 \max }\right\}, \\
& u_{3, k}^{*}=\min \left\{\max \left\{\frac{\left(\lambda_{2, k+1}-\lambda_{6, k+1}\right) \theta Q_{k}}{C_{k}}, u_{3 \min }\right\}, u_{3 \max }\right\} .
\end{aligned}
$$


Proof. The Hamiltonian $\widehat{H}_{k}$ at time step $k$ is given by

$$
\begin{aligned}
\widehat{H}_{k}= & H_{k}-T_{k}^{r}-T_{k}^{p}-Q_{k}+\frac{A_{k} u_{1, k}^{2}}{2}+\frac{B_{k} u_{2, k}^{2}}{2}+\frac{C_{k} u_{3, k}^{2}}{2} \\
& +\lambda_{1, k+1}\left[b-\beta_{1} \frac{P_{k} M_{k}}{N_{k}}+(1-\mu) P_{k}-u_{1, k} P_{k}\right] \\
& +\lambda_{2, k+1}\left[\beta_{1} \frac{P_{k} M_{k}}{N_{k}}+\left(1-u_{3, k}\right) \theta Q_{k}+\left(1-\mu-\beta_{2}\right) M_{k}\right] \\
& +\lambda_{3, k+1}\left[\beta_{2} M_{k}+\left(1-\mu-\delta-\alpha_{1}-\alpha_{2}-\alpha_{3}\right) H_{k}-u_{2, k} H_{k}\right] \\
& +\lambda_{4, k+1}\left[\alpha_{1} H_{k}+\left(1-\mu-\gamma_{1}\right) T_{k}^{r}+\epsilon u_{2, k} H_{k}\right] \\
& +\lambda_{5, k+1}\left[\alpha_{2} H_{k}+\left(1-\mu-\gamma_{2}\right) T_{k}^{p}+(1-\epsilon) u_{2, k} H_{k}\right] \\
& +\lambda_{6, k+1}\left[\gamma_{1} T_{k}^{r}+\alpha_{3} H_{k}+\gamma_{2} T_{k}^{p}+(1-\mu) Q_{k}+u_{1, k} P_{k}-\left(1-u_{3, k}\right) \theta Q_{k}\right] .
\end{aligned}
$$

For $k=0,1, \ldots, T-1$, the adjoint equations and transversality conditions can be obtained by using Pontryagin's maximum principle, in discrete time, given in [13-18] such that

$$
\begin{aligned}
& \lambda_{1, k}=\frac{\partial \widehat{H}_{k}}{\partial P_{k}}, \lambda_{1}(T) \\
& \lambda_{2, k}=\frac{\partial \widehat{H}_{k}}{\partial M_{k}}, \lambda_{2}(T) \\
& \lambda_{3, k}=\frac{\partial \widehat{H}_{k}}{\partial H_{k}}, \lambda_{3}(T) \\
& \lambda_{4, k}=\frac{\partial \widehat{H}_{k}}{\partial T_{k}^{r}}, \lambda_{4}(T) \\
& \lambda_{5, k}=\frac{\partial \widehat{H}_{k}}{\partial T_{k}^{p}}, \lambda_{5}(T) \\
& \lambda_{6, k}=\frac{\partial \widehat{H}_{k}}{\partial Q_{k}}, \lambda_{6}(T)
\end{aligned}
$$

For $k=0,1, \ldots, T-1$, the optimal controls $u_{1, k}^{*}, u_{2, k}^{*}$, and $u_{3, k}^{*}$ can be solved from the optimality condition

$$
\begin{aligned}
& \frac{\partial \widehat{H}_{k}}{\partial u_{1, k}}=A_{k} u_{1, k}-\lambda_{1, k+1} P_{k}+\lambda_{6, k+1} P_{k}=0, \\
& \frac{\partial \widehat{H}_{k}}{\partial u_{2, k}}=B_{k} u_{2, k}-\lambda_{3, k+1} H_{k}+\lambda_{4, k+1} \epsilon H_{k}+\lambda_{5, k+1}(1-\epsilon) H_{k}=0, \\
& \frac{\partial \widehat{H}_{k}}{\partial u_{3, k}}=C_{k} u_{3, k}-\lambda_{2, k+1} \theta Q_{k}+\lambda_{6, k+1} \theta Q_{k}=0 .
\end{aligned}
$$

So, we have

$$
\begin{aligned}
& u_{1, k}=\frac{\left(\lambda_{1, k+1}-\lambda_{6, k+1}\right) P_{k}}{A_{k}}, \\
& u_{2, k}=\frac{\left(\lambda_{3, k+1}-\lambda_{5, k+1}\right) H_{k}+\left(\lambda_{5, k+1}-\lambda_{4, k+1}\right) \epsilon H_{k}}{B_{k}}, \\
& u_{3, k}=\frac{\left(\lambda_{2, k+1}-\lambda_{6, k+1}\right) \theta Q_{k}}{C_{k}} .
\end{aligned}
$$

By the bounds in $U_{\text {ad }}$ of the controls, it is easy to obtain $u_{1, k}^{*}, u_{2, k}^{*}$, and $u_{3, k}^{*}$ in the form of (10).

\section{Numerical Simulation}

In this section, we shall solve numerically the optimal control problem for our $P M H T^{r} T^{P} Q$ model. Here, we obtain the optimality system from the state and adjoint equations. The proposed optimal control strategy is obtained by solving the optimal system which consists of six difference equations and boundary conditions. The optimality system can be solved by using an iterative method. Using an initial guess for the control variables, $u_{1, k}, u_{2, k}$, and $u_{3, k}$, the state variables, $P, M, H, T^{r}, T^{p}$, and $Q$, are solved forward and the adjoint variables $\lambda_{i}$ for $i=1,2,3,4,5,6$ are solved backwards at time steps $k=0$ and $k=T$. If the new values of the state and adjoint variables differ from the previous values, the new values are used to update $u_{1, k}, u_{2, k}$, and $u_{3, k}$, and the process is repeated until the system converges.

The numerical solution of model (1) is executed using Matlab with the following parameter values and initial values of state variable in Table 1.

We begin by presenting the solution evolution of our model (1) without controls that are represented in Figure 2. 
Figure 2(a) shows that the number of moderate drinkers increases in the beginning but then decreases clearly. This decrease leads to an increase in the number of heavy drinkers from the beginning and approaches a given value of 462.30 as we see in Figure 2(b). Finally, Figure 2(c) and Figures 2(d) and $2(\mathrm{e})$ show that the number of the rich and poor heavy drinkers joining private and public centers for alcohol addiction treatment and the quitters of drinking decreases and approaches certain unacceptable values.

In order to display the influence of alcohol treatment centers on the population drinking dynamics, we present some figures about the effects of the parameters $\alpha_{1}, \alpha_{2}, \gamma_{1}$, and $\gamma_{2}$ on system (1).

Figure 2(f) shows that the number of heavy drinkers increases when the number of the heavy drinkers who join addiction treatment centers decreases. Also, we observe in Figure 2(g) that the number of quitters of drinking increases when the number of the heavy drinkers who join addiction treatment centers increases.

From these two figures, the importance of addiction treatment centers is clear in reducing the number of heavy drinkers and thus increasing the number of quitters of drinking. Therefore, we need to make an effort to introduce these centers to people and encourage heavy drinkers to join them and this is shown through the following objectives.

5.1. First Objective: Protecting and Preventing Potential Drinkers from Falling into Alcohol Addiction. To realize this objective, we apply only the control $u_{1}$, i.e., the implementation of awareness, information, and educational programs on potential drinkers and making them know the risks of this phenomenon and the resulting health and social damages. Figure 3(a) shows that the number of moderate drinkers decreases from 231.16 (without control $u_{1}$ ) to 124.59 (with control $u_{1}$ ) at the end of the proposed program and the number of heavy drinkers decreases from 462.29 (without control $u_{1}$ ) to 249.02 (with control $u_{1}$ ) at the end of the proposed control strategy (see Figure 3(b)). Also, we observe in Figure 3(c) that the number of people who quit drinking increases and has reached the value 429.78 (with control $u_{1}$ ) compared to the situation when there is no control, 5.68, at the end of the proposed strategy. So, our objective has been achieved.

5.2. Second Objective: Increasing the Number of Heavy Drinkers Who Join Treatment Centers of Alcohol Addiction. To achieve this objective, we only use the control $u_{2}$, i.e., encouraging heavy drinkers to know and join public and private treatment centers. In Figure 4(a) it is observed that there is a significant decrease in the number of heavy drinkers with control compared to a situation when there is no control where the decrease reaches $41.20 \%$ at the end of the proposed control strategy. Figure $4(\mathrm{~b})$ shows that the number of the poor heavy drinkers who join public treatment centers increased from $6.91\left(T^{p}\right.$ without control $u_{2}$ ) to 59.70 ( $T^{p}$ with control $u_{2}$ ) and that the number of rich heavy drinkers who join private treatment centers increased from 7.06 (without control $u_{2}$ ) to 233.72 (with control $u_{2}$ ) at the
TABle 1: The parameters used for model (1).

\begin{tabular}{lcccccccc}
\hline$P_{0}$ & $M_{0}$ & $H_{0}$ & $T_{0}^{r}$ & $T_{0}^{p}$ & $Q_{0}$ & $b$ & $N$ & $\delta$ \\
500 & 300 & 100 & 60 & 30 & 10 & 65 & 1000 & 0.002 \\
\hline$\mu$ & $\beta_{1}$ & $\beta_{2}$ & $\alpha_{1}$ & $\alpha_{2}$ & $\alpha_{3}$ & $\gamma_{1}$ & $\gamma_{2}$ & $\theta$ \\
0.065 & 0.75 & 0.14 & 0.001 & 0.001 & 0.001 & 0.001 & 0.002 & 0.02 \\
\hline
\end{tabular}

end of the proposed control (see Figure 4(c)) and Figure 4(d) shows that the number of people who quit drinking without control $u_{2}$ decreases and approaches a value of 5.68. After 5 days, a slight increase appears with control $u_{2}$. This increase is not enough as the number of people who quit drinking becomes moderate drinkers. To improve this result, we can add another control that represents follow-up for temporary quitters of drinking.

\subsection{Third Objective: Encouraging Heavy Drinkers to Join} Treatment Centers of Alcohol Addiction and Following Up the Quitters of Drinking. In order to accomplish this aim, we use the controls $u_{2}$ and $u_{3}$, i.e., encouraging heavy drinkers to know and join public and private treatment centers and following up the temporary quitters of drinking. The number of the poor heavy drinkers who join public treatment centers increases from 6.91 (without controls) to 32.17 (with controls) at the end of the proposed strategy (see Figure 5(c)). Figure 5(d) demonstrates that the number of the rich heavy drinkers who join private treatment centers increases from 7.06 (without controls) to 125.92 (with controls) at the end of the proposed strategy. The number of the quitters of drinking in Figure 5(e) increases significantly from 5.68 (without controls) to 430.11 (with controls). Through the use of those controls, the abovementioned objective has been achieved.

5.4. Fourth Objective: Prevention, Treatment, and Follow-Up for the Addicted Individuals. To meet this objective, we use the controls $u_{1}, u_{2}$, and $u_{3}$, i.e., awareness programs for the potential drinkers, encouraging heavy drinkers to know and join public and private treatment centers, and follow-up for the quitters of drinking. Figure 6(a) shows that the number of the moderate drinkers decreases starting from the early days by $34.60 \%$. Also, Figure 6(b) shows that the number of the heavy drinkers increases starting from the early days but then decreases from 462.29 (without controls) to 65.81 (with controls). The number of the rich and poor heavy drinkers who join private and public treatment centers increases by $11.45 \%$ and $2.98 \%$, respectively (see Figures 6(c) and 6(d)). Figure $6(\mathrm{e})$ depicts clearly an increase in the number of the quitters of drinking from 5.68 (without controls) to 550.63 (with controls). As a result, the objective set before has been achieved.

We remark that the numbers of the rich and poor heavy drinkers $\left(T^{r}\right.$ and $\left.T^{p}\right)$ decrease when we applied three controls $\left(u_{1}, u_{2}, u_{3}\right)$ compared to using only the control $\left(u_{2}\right)$. This decrease is due to the positive effect of the control $u_{1}$ on the compartment $P$ and the control $u_{3}$ on $\theta Q$ (temporary quitters) which prevents temporary quitters of 


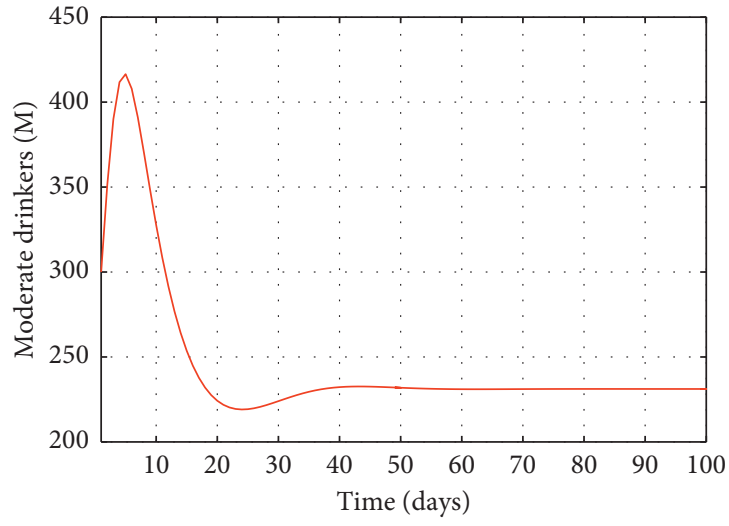

- $M$ without controls

(a)

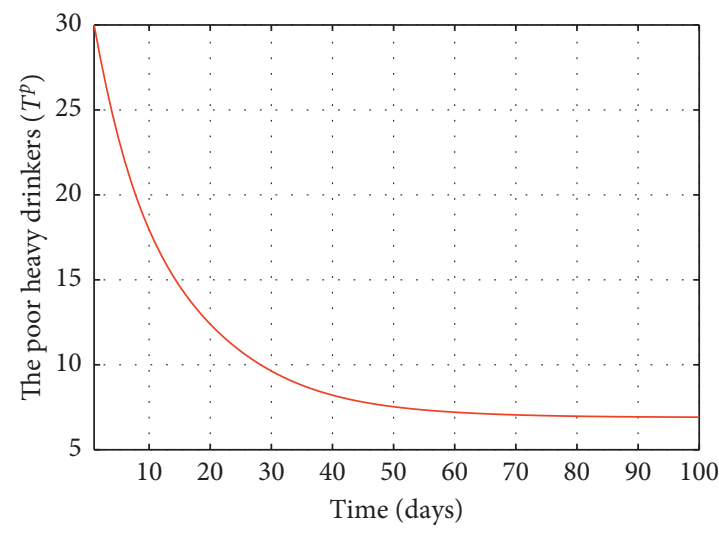

— $T^{p}$ without controls

(c)

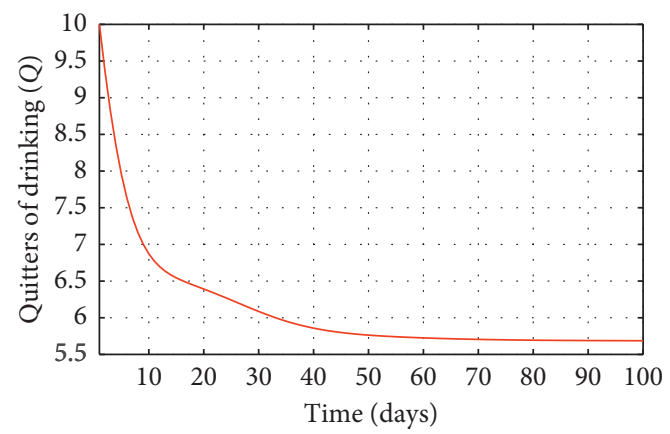

Q without controls

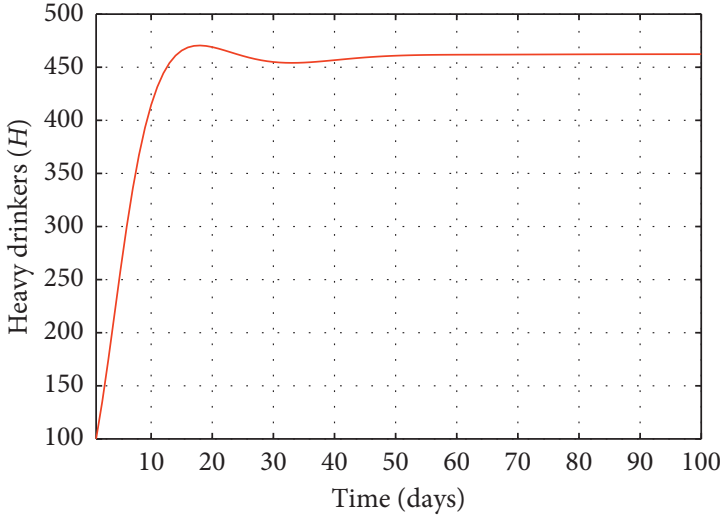

— $H$ without controls

(b)

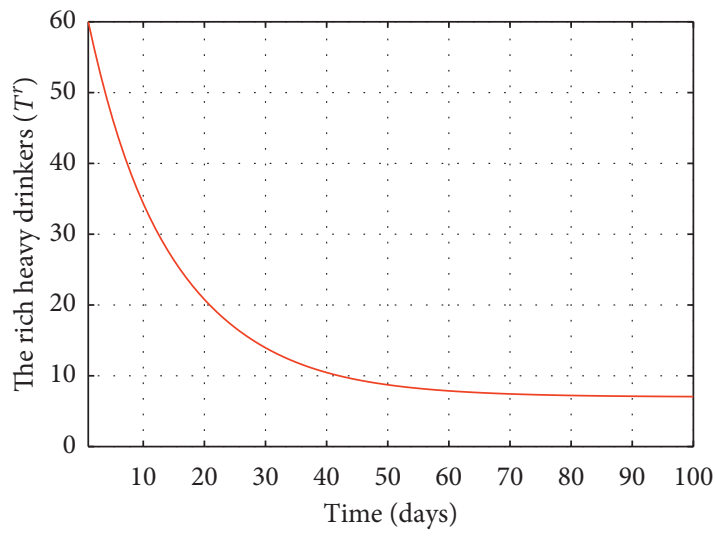

— $T^{r}$ without controls

(d)

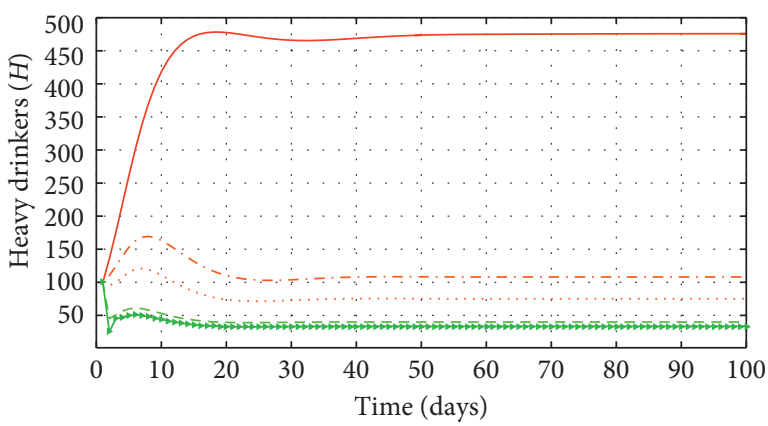

- $H$ without treatment centers of alcohol addiction

... $H$ with $\alpha_{1}=0.2 ; \alpha_{2}=0.05 ; \gamma_{1}=0.04 ; \gamma_{2}=0.02$

$H$ with $\alpha_{1}=0.3 ; \alpha_{2}=0.1 ; \gamma_{1}=0.06 ; \gamma_{2}=0.03$

- - $H$ with $\alpha_{1}=0.6 ; \alpha_{2}=0.3 ; \gamma_{1}=0.5 ; \gamma_{2}=0.2$

$\rightarrow H$ with $\alpha_{1}=0.7 ; \alpha_{2}=0.4 ; \gamma_{1}=0.6 ; \gamma_{2}=0.3$

(e)

(f)

FIgURe 2: Continued. 


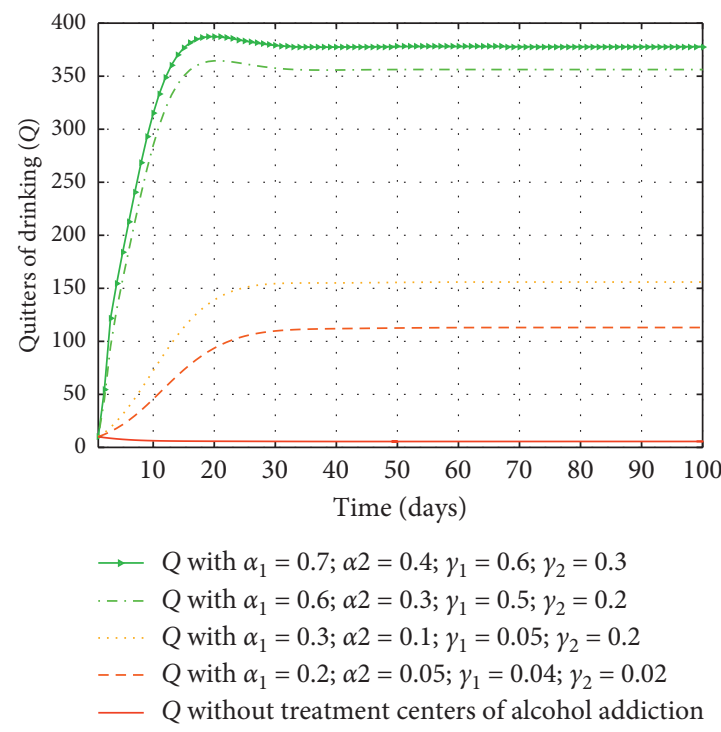

(g)

FIGURE 2: Representing the drinkers class without control.
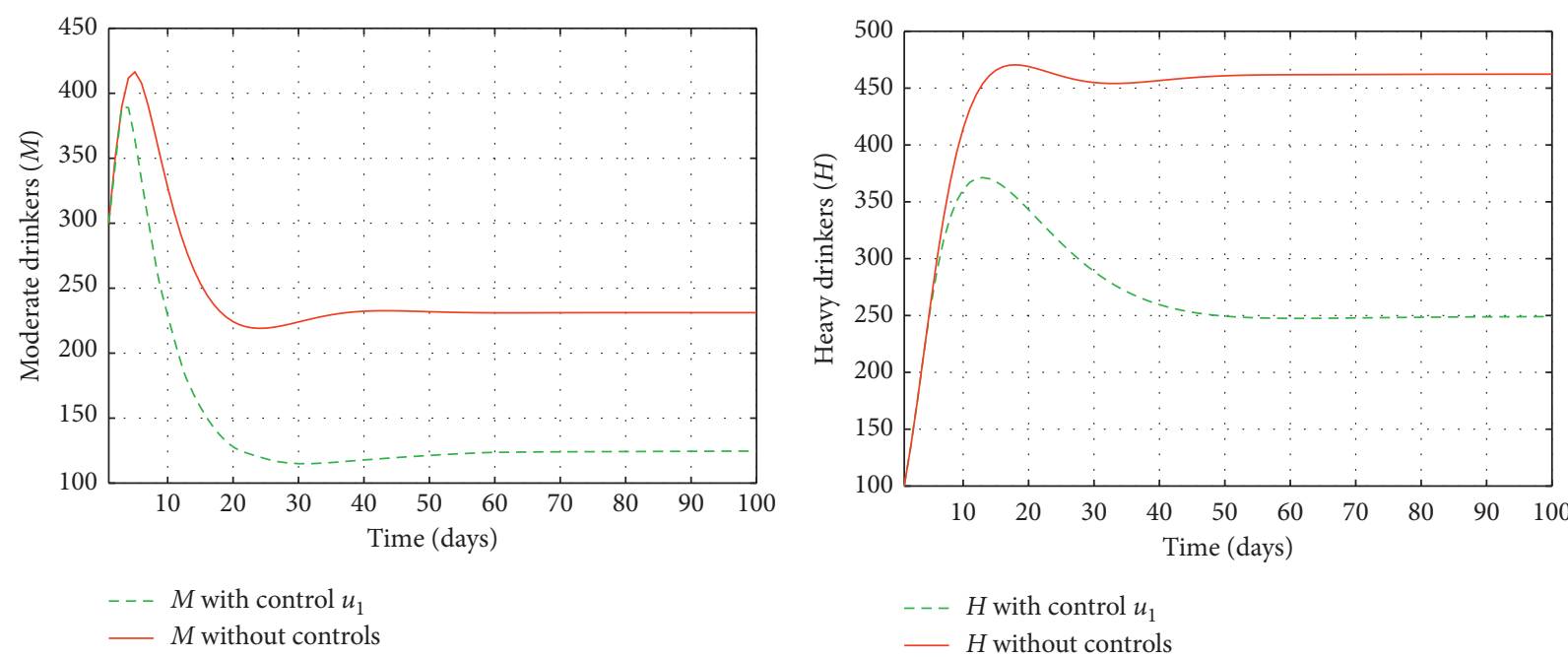

(a)

(b)

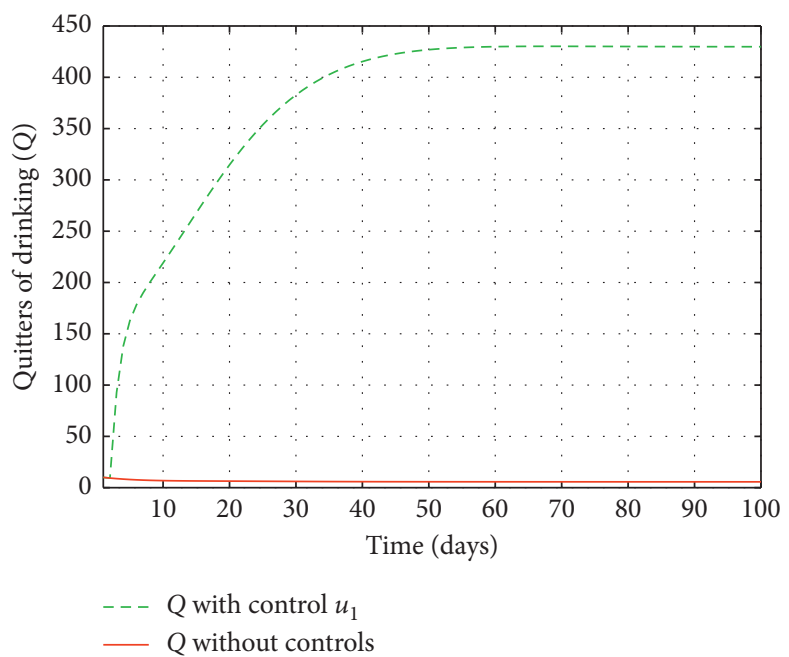

(c)

FIgURE 3: Representing the drinkers class with and without control $u_{1}$. 


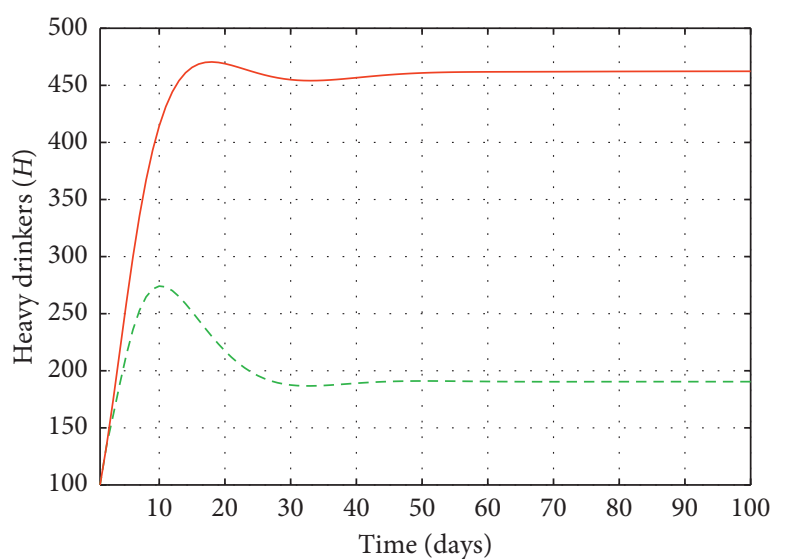

- - $H$ with control $u_{2}$

— $H$ without controls

(a)

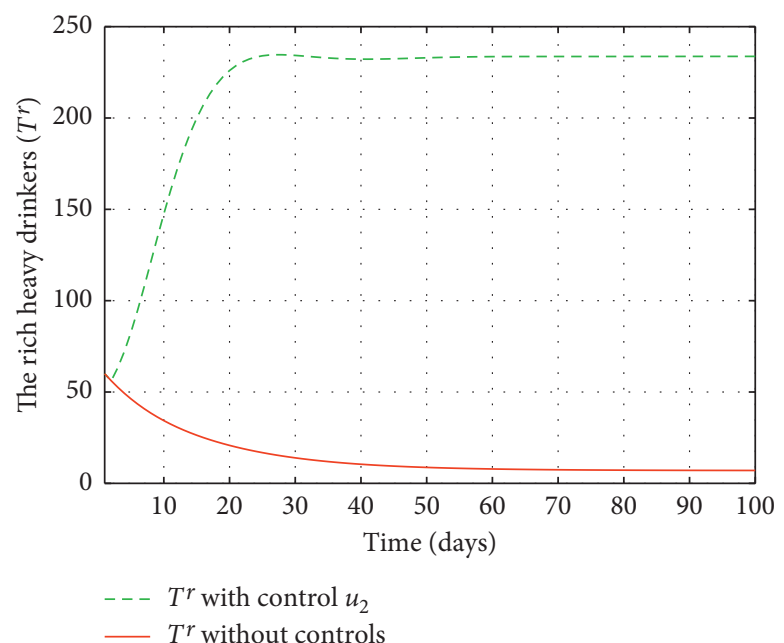

(c)

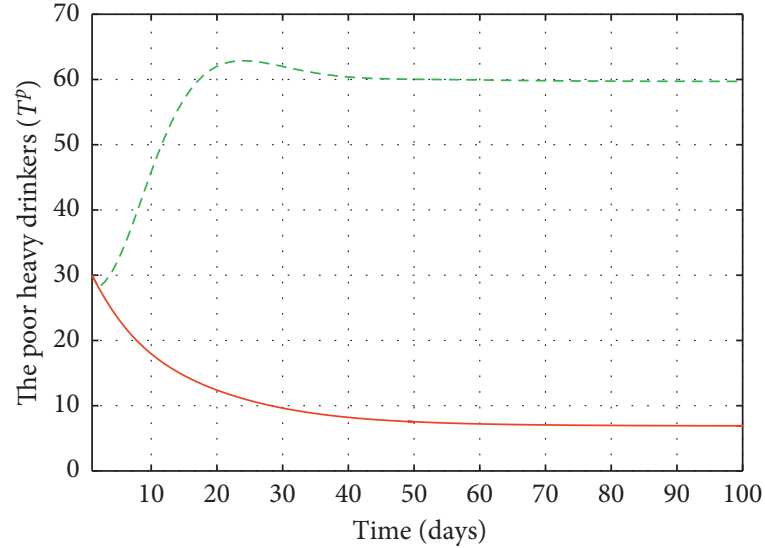

-.- $T^{p}$ with control $u_{2}$

- $T^{p}$ without controls

(b)

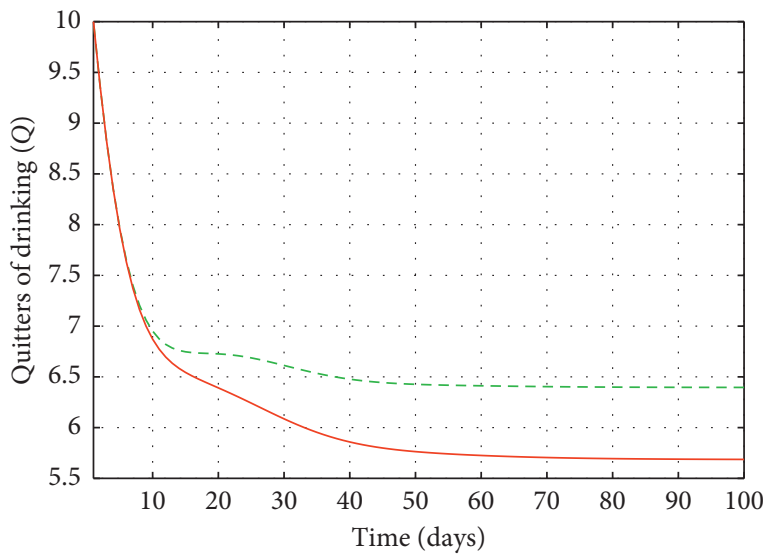

- - Q $Q$ with control $u_{2}$

- $Q$ without controls

Figure 4: Representing the drinkers class with and without control $u_{2}$.

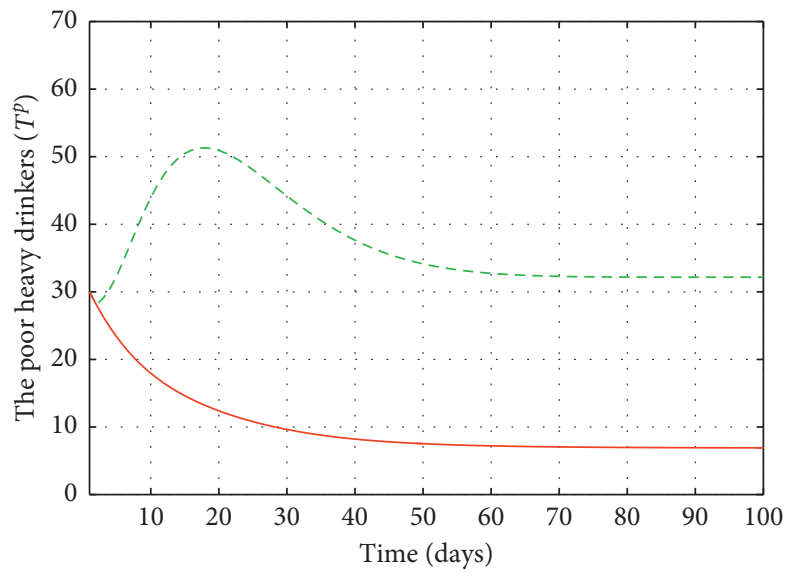

- - $T^{p}$ with controls $u_{2}$ and $u_{3}$

— $T^{p}$ without controls

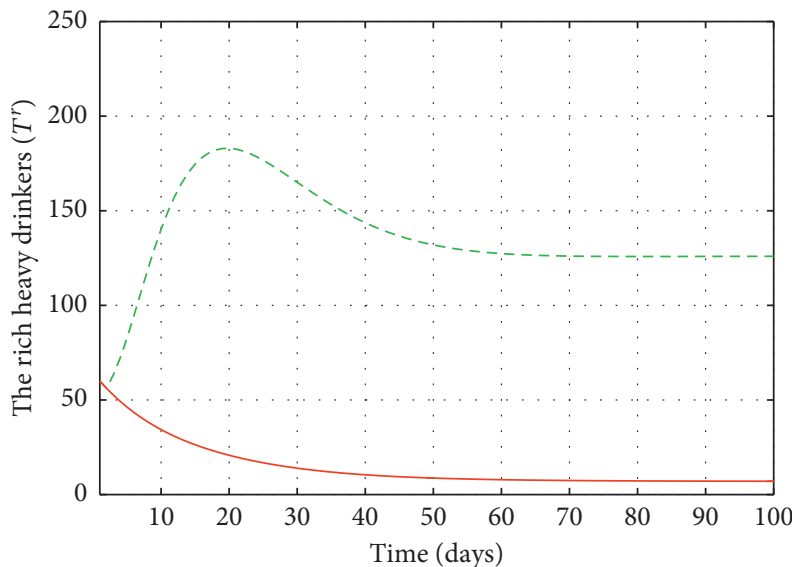

- - $T^{r}$ with controls $u_{2}$ and $u_{3}$

— $T^{r}$ without controls

(a)

Figure 5: Continued. 


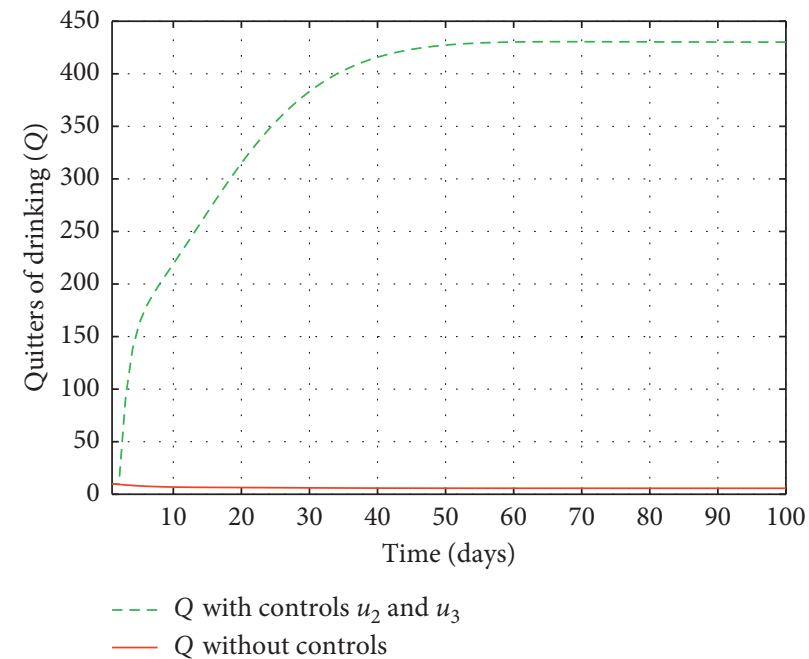

(c)

FIGURE 5: Representing the drinkers class with controls $u_{2}$ and $u_{3}$ and without controls.

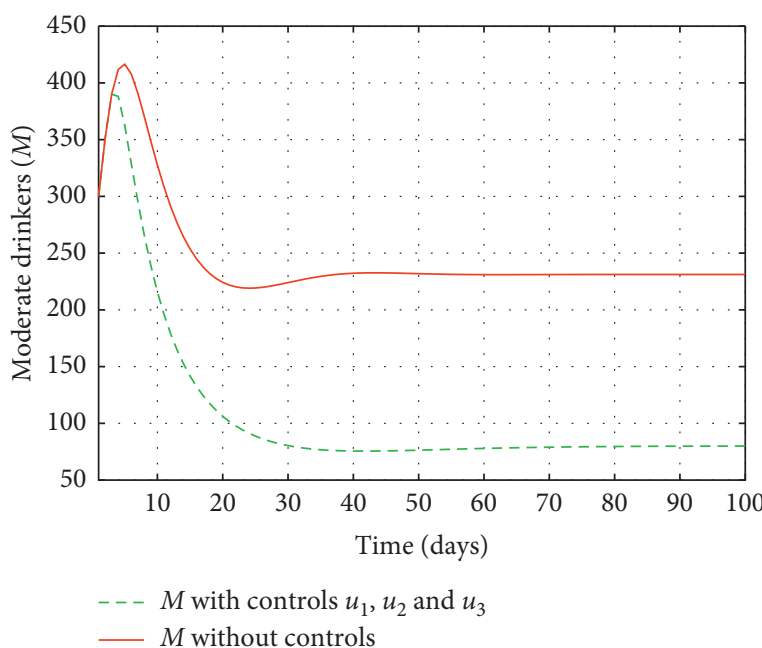

(a)

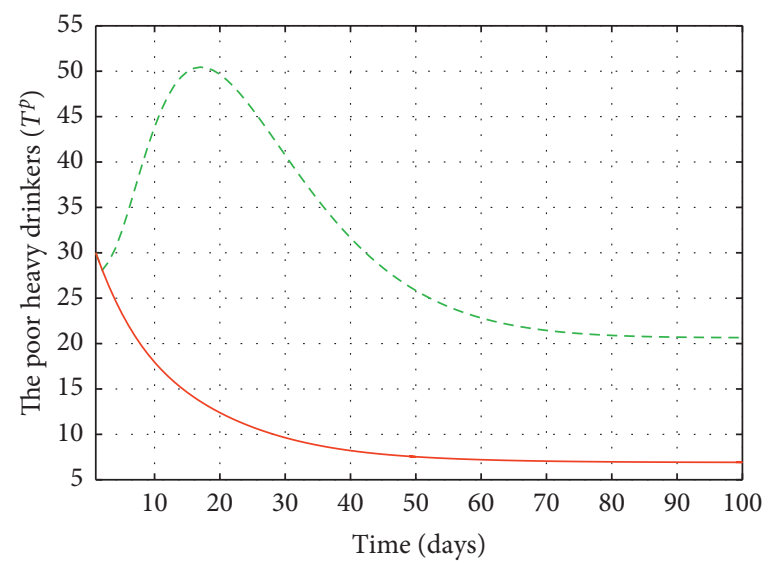

- - $T^{p}$ with controls $u_{1}, u_{2}$ and $u_{3}$ $T^{p}$ without controls

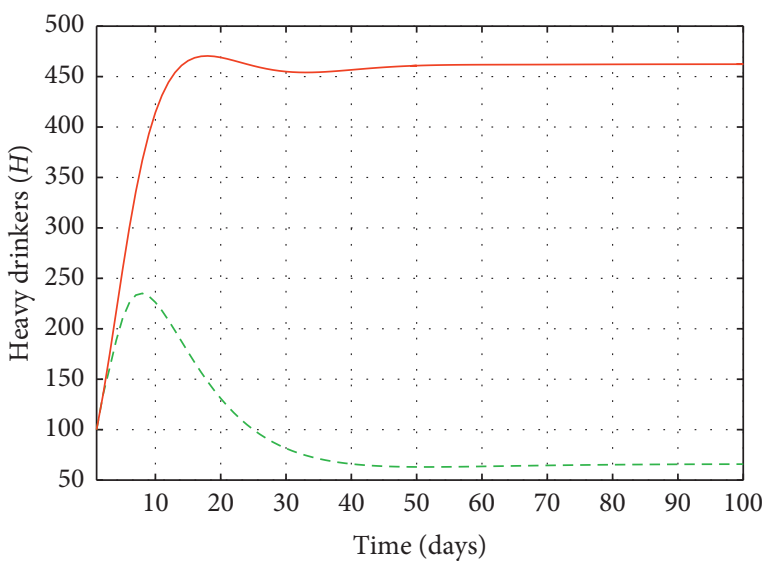

- - $H$ with controls $u_{1}, u_{2}$ and $u_{3}$ - $H$ without controls

(b)

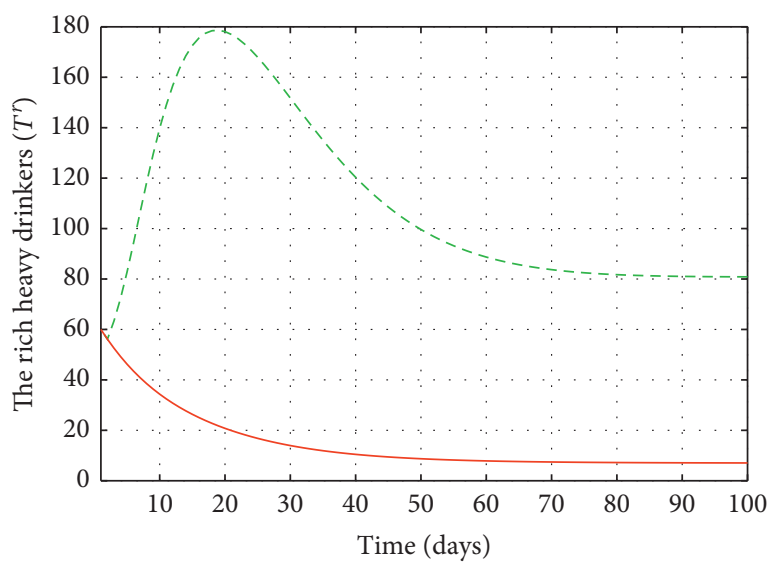

-- $T^{r}$ with controls $u_{1}, u_{2}$ and $u_{3}$ $T^{r}$ without controls

(c)

Figure 6: Continued. 


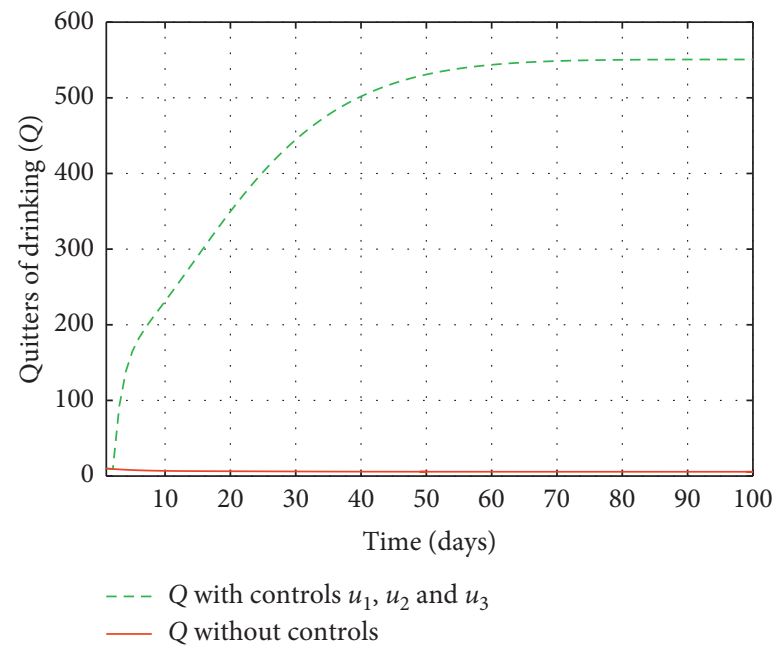

(e)

Figure 6: Representing the drinkers class with and without optimal controls $u_{1}, u_{2}$, and $u_{3}$.

drinking from returning to drinking. So, the number of individuals that enter the compartment, $T^{r}$ and $T^{p}$, decreases. Therefore, applying a combination of the three controls is more effective than one control.

\section{Conclusion}

In this research paper, we introduced a discrete modeling of drinking for the purpose of minimizing the number of drinkers and maximizing the number of the rich and poor heavy drinkers who join private and public treatment centers of alcohol addiction and subsequently the number of quitters of drinking. Unlike some other previous models, we have taken into account the impact of private and public addiction treatment centers on alcoholics. The results showed that those centers have substantial influence on the dynamics of alcoholism and can greatly impact the spread of drinking. Thus, it is crucial to urge people to know and join private and public addiction treatment centers to quit drinking. We also presented three controls which, respectively, represent awareness programs, encouragement, and follow-up. We applied the results of the control theory and we managed to obtain the characterizations of the optimal controls. The numerical simulation of the obtained results showed the effectiveness of the proposed control strategies.

\section{Data Availability}

The disciplinary data used to support the findings of this study have been deposited in the Network Repository (http://networkrepository.com/profile.php).

\section{Conflicts of Interest}

The authors declare that there are no conflicts of interest regarding the publication of this paper.

\section{References}

[1] WHO, World Health Organization Report on Drinking, WHO, Geneva, Switzerland, 2016.

[2] World Health Organization, Alcohol, World Health Organization, Geneva, Switzerland, 2018, https://www.who.int/ news-room/fact-sheets/detail/alcohol.

[3] H. F. Huo and Q. Wang, "Modelling the influence of awareness programs by media on the drinking dynamics," Abstract and Applied Analysis, vol. 2014, Article ID 938080, 8 pages, 2014.

[4] S.-H. Ma, H.-F. Huo, and X.-Y. Meng, "Modelling alcoholism as a contagious disease: a mathematical model with awareness programs and time delay," Discrete Dynamics in Nature and Society, vol. 2015, Article ID 260195, 13 pages, 2015.

[5] X.-Y. Wang, H.-F. Huo, Q.-K. Kong, and W.-X. Shi, "Optimal control strategies in an alcoholism model," Abstract and Applied Analysis, vol. 2014, Article ID 954069, 18 pages, 2014.

[6] H.-F. Huo, S.-R. Huang, X.-Y. Wang, and H. Xiang, "Optimal control of a social epidemic model with media coverage," Journal of Biological Dynamics, vol. 11, no. 1, pp. 226-243, 2017.

[7] H. F. Huo and N. N. Song, "Global stability for a binge drinking model with two stages," Discrete Dynamics in Nature and Society, vol. 2012, Article ID 829386, 15 pages, 2012.

[8] I. K. Adu, M. Osman, and C. Yang, "Mathematical model of drinking epidemic," British Journal of Mathematics Computer Science, vol. 22, no. 5, pp. 1-10, 2017.

[9] H.-F. Huo and Y.-P. Liu, "The analysis of the SIRS alcoholism models with relapse on weighted," SpringerPlus, vol. 5, no. 1, p. 722, 2016.

[10] K. Mu'tamar, "Optimal control strategy for alcoholism model with two infected compartments," IOSR Journal of Mathematics, vol. 14, no. 3, pp. 58-67, 2018.

[11] S. Sharma and G. P. Samanta, "Drinking as an epidemic: a mathematical model with dynamic behaviour," Journal of Applied Mathematics \& Informatics, vol. 31, no. 1-2, pp. 1-25, 2013.

[12] S. Sharma and G. P. Samanta, "Analysis of a drinking epidemic model," International Journal of Dynamics and Control, vol. 3, no. 3, pp. 288-305, 2015. 
[13] W. Ding, R. Hendon, B. Cathey, E. Lancaster, and R. Germick, "Discrete time optimal control applied to pest control problems," Involve, A Journal of Mathematics, vol. 7, no. 4, pp. 479-489, 2014.

[14] V. Guibout and A. M. Bloch, "A discrete maximum principle for solving optimal control problems," in Proceedings of the 2004 43rd IEEE Conference on Decision and Control (CDC), vol. 2, pp. 1806-1811, Nassau, Bahamas, December 2004.

[15] C. L. Hwang and L. T. Fan, "A discrete version of pontryagin's maximum principle,” Operations Research, vol. 15, no. 1, pp. 139-146, 1967.

[16] L. S. Pontryagin, V. G. Boltyanskii, R. V. Gamkrelidze, and E. F. Mishchenko, The Mathematical Theory of Optimal Processes, John Wiley \& Sons, London, UK, 1962.

[17] S. Lenhart and J. T. Workman, Optimal Control Applied to Biological Models, Chapman \& Hall/CRC, Boca Raton, FL, USA, 2007.

[18] D. C. Zhang and B. Shi, "Oscillation and global asymptotic stability in a discrete epidemic model," Journal of Mathematical Analysis and Applications, vol. 278, no. 1, pp. 194-202, 2003. 